\title{
CORRESPONDENCE
}

\section{Beyond evidence-based Medicine}

\author{
A.M. Abeygunasekera \\ Colombo South Teaching Hospital, Sri Lanka
}

It is with interest that I read the editorial titled evidence-based practice, a must in medical practice [1]. While agreeing with all the reasons given to promote evidence based practice among surgeons, I would like to highlight certain shortcomings in evidence-based medicine (EBM) which persuades me to propose that we should move beyond the concept of EBM to a better paradigm which is more appropriate for the delivery of good health care in Sri Lanka.

Several decades ago the necessity for EBM was obvious. At that time medical personnel used to practice ego-based medicine where the doctor said "I know everything, I can never go wrong, my word is the last, you do what I say, there is no need for questions or critical analysis". Those who realised the perils of ego-based medicine found this new paradigm of evidence-based medicine to improve patient care. EBM was an important and necessary step forward from ego-based medicine. Therefore the medical world embraced EBM as the new paradigm. EBM was more scientifically appealing than ego-based medicine and helped to improve the health care tremendously. Even today it is a better alternative to ego-based medicine as highlighted in the editorial [1].

However after practicing EBM for several decades I have encountered some deficiencies of EBM. Initially EBM was defined as conscientious, explicit and judicious use of current best evidence in making decisions about the care of individual patients. Later another phrase was added to make it more realistic - integrating individual clinical expertise and the best external evidence. However those who practice medicine in the real life situation know that these idealistic words in the EBM definition are only a utopian dream. In reality such perfect health care is so expensive and demanding that it can be provided only to a handful of the society especially in resource poor settings prevalent in developing countries. Medical personnel who try to provide reasonable health care to all members of the society will be reprimanded for ignoring EBM in their practice. Therefore EBM has become a catch word that is abused by those who want to provide 'perfect' health care to a few privileged patients in the society. It has also given a 'scientific back-up' to those in the industry who want to sell their expensive products (with marginal or no benefits to the patient) for personal gains and to make exorbitant profits.

Publication bias, language bias, support for research from the pharmaceutical industry and lack of research in the local settings of the developing world are some of the obstacles faced during the search for current best evidence. Cultural differences, social differences and cost effectiveness make the issue more complex. Cost effectiveness which is crucial for the sustenance of any health care system is greatly ignored in EBM as there is hardly any research on cost effectiveness of therapeutic interventions in the developing world. This has lead to diversion of limited resources available in developing countries to relatively expensive interventions as medical personnel are forced to endorse such processes based on evidence generated in the developed world. Failure to do so may lead to allegations of malpractice and litigation. Guidelines and protocols based on EBM are abused by parties interested in making money out of litigation. Therefore EBM promotes defensive medicine.

Hence time is opportune for the medical community to find a new paradigm in delivery of health care which would rectify the deficiencies of the concept of EBM when practiced in resource poor countries and settings. The main aim of the new paradigm would be to provide reasonable health care to all. This could be appropriately called equity-based medicine. At present there is no political and administrative will or leadership to appreciate and support those who provide reasonable health care to all segments of the society in resource poor settings. Politicians and administrators are also misguided and frightened by the concept of EBM and have no courage to contest the inappropriateness of EBM in

The Sri Lanka Journal of Surgery 2014; 32(2): 53-54 
resource poor settings.

We need more research on cost effectiveness done at the point of delivery. Guidelines should take into consideration the situation in the local setting. Politicians, administrators and decision makers should be encouraged to give leadership to low cost health care and reasonable care to all rather than ideal care to a few. Changes should be made in the legal framework to accept equity-based medicine as the new paradigm. We changed from ego-based medicine to evidencebased medicine two decades ago. Now it is time to improve evidence-based medicine and step into equity-based medicine.

\section{References}

1. Abeygunaratne Ruvini. Evidence-based practice, a must in medical practice. Sri Lanka Journal of Surgery 2014;32: 1-2 